\title{
Gravity and the Absoluteness of Time: a Simple Qualitative Model
}

\author{
Carmine Cataldo ${ }^{1}$ \\ ${ }^{1}$ Independent Researcher, $\mathrm{PhD}$ in Mechanical Engineering, Battipaglia (Salerno), Italy \\ Correspondence: Carmine Cataldo, Battipaglia (Salerno), Italy. E-mail: catcataldo@hotmail.it
}

Received: February 1, 2017

Accepted: February 24, 2017

Online Published: March 7, 2017

doi:10.5539/apr.v9n2p42

URL: https://doi.org/10.5539/apr.v9n2p42

\begin{abstract}
In this paper, we qualitatively examine the compatibility between gravity and the absoluteness of time. Initially, time is supposed as being absolute. However, this assumption does not imply that instruments and devices, finalized to measure time, are not influenced by gravity. On the contrary, we admit that whatever phenomenon, including the ones that occur when we measure time, shows clear traces of the influence of gravity. Nonetheless, the alleged time dilation, that seems to occur when we approach a gravitational source, could actually be illusory. In this paper, in fact, we contemplate the possibility that the above-mentioned phenomenon could be exclusively related to the contraction of the orbits induced by the mass that produces the gravitational field. We start from postulating a Universe, belonging to the oscillatory class, characterized by at least a further spatial dimension. At the beginning, the Universe in its entirety is assimilated to a four-dimensional ball, and matter is considered as being evenly spread. Once hypothesized that all the available mass may be concentrated in a single point, taking advantage of an opportune parameterization, pretending that the orbits don't undergo any modification whatsoever and admitting, as a consequence, that time starts slowing down when we move towards the singularity, we can easily obtain, far from the source, a Schwarzschild solution for the vacuum field, without using General Relativity.
\end{abstract}

Keywords: Gravitation; Gravitational Singularity, General Relativity, Vacuum Field, Weak Field Approximation, Schwarzschild Metric, Absoluteness of Time

\section{Introduction}

We hypothesize a closed Universe belonging to the oscillatory class (Harrison, 1967). More precisely, we postulate a Universe that evolves following a simple harmonic motion whose pulsation is equal to the ratio between the speed of light and the mean radius (of curvature). The existence of at least a further spatial dimension is contemplated. Although the space we are allowed to perceive is curved, since it is identifiable with a hyper-sphere whose radius depends on our state of motion, the Universe in its entirety, herein assimilated to a four-dimensional ball, is to be considered as being flat (Cataldo, 2017). All the points are replaced by straight line segments: in other terms, what we perceive as being a point is actually a straight-line segment crossing the centre of the abovementioned four-dimensional ball (Cataldo, 2016a).

If $R$ represents the radius, the Universe we have hypothesized can be banally described, with obvious meaning of the notation, by means of the following inequality:

$$
x_{1}^{2}+x_{2}^{2}+x_{3}^{2}+x_{4}^{2} \leq R^{2}
$$

However, the Universe we are allowed to perceive, when we are at rest, can be assimilated to the hyper-surface defined by the underlying identity:

$$
x_{1}^{2}+x_{2}^{2}+x_{3}^{2}+x_{4}^{2}=R^{2}
$$

In a closed Universe, isotropic and homogeneous, matter is usually imagined as being evenly spread on the surface of a four-dimensional ball. In our case, we actually postulate that matter fills the 4-ball in its entirety, although the Universe we are allowed to perceive can be assimilated to a hypersphere. Let's denote with $C$ the centre of the 4ball, with $O$ and $P$ two points on the surface, the first of which taken as origin, and with $O$ ' the centre of the socalled measured circumference, to which $P$ belongs. The angular distance between $O$ and $P$, as perceived by an ideal observer placed in $C$, is denoted by $\chi$. 
The circumference, whose center coincides with $O^{\prime}$, is simultaneously boundary of the disk, whose radius is equal to the forecast one, and of the spherical cap, whose amplitude is equal to $2 \chi$. Similarly, the spherical surface, whose center coincides with $O^{\prime}$, is simultaneously boundary of the ball, whose radius is equal to the forecast one, and of the hyper-spherical cap (a three-dimensional surface), whose amplitude is equal to $2 \chi$, characterized by a volume, denoted by $V$, provided by the following identity:

$$
V=\int_{0}^{\chi} 4 \pi(R \sin \chi)^{2} R d \chi=2 \pi R^{3}(\chi-\sin \chi \cos \chi) \cong \frac{4}{3} \pi \chi^{3} R^{3}
$$

The "flat coordinate" (the forecast radius), denoted by $X$, is provided by the following relation:

$$
X=R \sin \chi
$$

If we denote with $l$ the measured distance (the proper radius), we can write:

$$
d l=R d \chi=\frac{d X}{\sqrt{1-\left(\frac{X}{R}\right)^{2}}}
$$

Once introduced the dimensionless coordinate provided by the underlying identity

$$
\bar{r}=\frac{X}{R}=\sin \chi
$$

we can write, with obvious meaning of symbols and signs, the well-known Friedmann - Robertson - Walker metric, with the scale (dimensional) factor equal to $R$ :

$$
d s^{2}=c^{2} d t^{2}-R^{2}\left[\frac{d \bar{r}^{2}}{1-\bar{r}^{2}}+\bar{r}^{2}\left(d \theta^{2}+\sin ^{2} \theta d \varphi^{2}\right)\right]
$$

The situation is qualitative depicted in Figure 1.

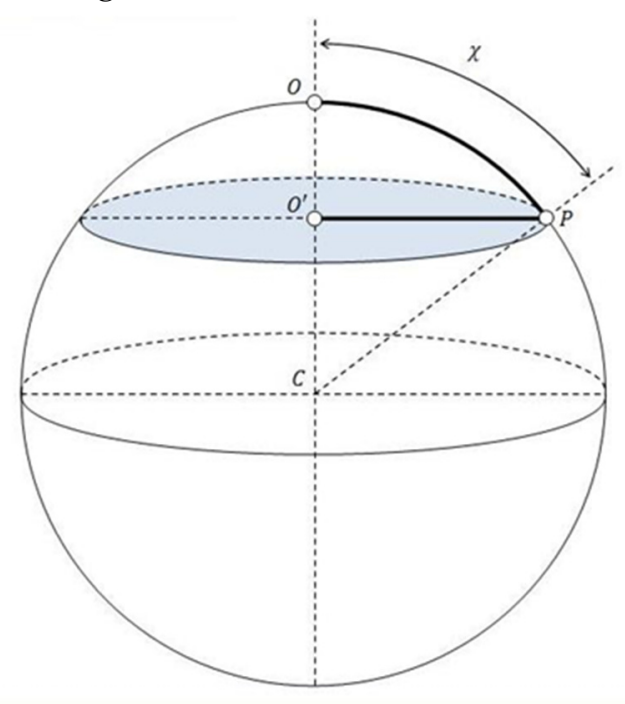

Figure 1. Forecast Radius and Measured Distance

Figure 1 shows the difference between the forecast radius and the measured distance. The straight-line segment bordered by $O$ ' and $P$ represents the forecast radius (that can be expressed as the ratio between the perimeter of the measured circumference and $2 \pi$ ). The arc bordered by $O$ and $P$ represents the proper radius (the measured distance between the above-mentioned points).

\section{How Matter warps Space: a simple qualitative model}

In deducing the so-called Friedmann - Lemaître equations (Friedmann 1922), having hypothesized an isotropic and homogeneous Universe that oscillates simple-harmonically, we have elsewhere carried out the following noteworthy position (Cataldo, 2017): 


$$
c^{2}=\frac{2 G M_{m}}{R_{m}}
$$

$G$ represents the gravitational constant, $c$ the speed of light, $R_{m}$ the mean value of the radius (of curvature) of the Universe, and $M_{m}$ the correspondent mass. It is fundamental to highlight how the values of $R_{m}$ and $M_{m}$ must be conventionally considered as being the real ones, since the metric variation of cosmological distances is not thought to be a real phenomenon (in other terms, the amount of space between whatever couple of points remains constant with the passing of time). From the previous equation, denoting with $R_{s}$ the so-called Schwarzschild radius, and replacing, for convenience, $M_{m}$ with $M$, we can immediately write:

$$
R_{m}=\frac{2 G M}{c^{2}}=R_{S}(M)
$$

From now onwards, we will exclusively speak in terms of alleged real values (of distances): as a consequence, $R$ will always be replaced by $R_{s}$.

Let's consider now a generic point $Q$, belonging to the surface of the 4-ball, and let's denote with $\chi_{\text {max }}$ the angular distance between this point and the origin. We have now to suppose that the mass contained in the hyper-spherical sector, whose amplitude is equal to $2 \chi_{\max }$, may be evenly spread along the segment bordered by $C$ and $O$ (perceived as being a material point), so producing an increment of its linear density. It follows that the segment undergoes a radial contraction: given that its mass increases, the new amount of energy is no longer compatible with the previous radial extension. To even only qualitatively explain this, we cannot exploit the alternative writing of the conservation of energy elsewhere discussed (Cataldo, 2016b). In fact, since its linear density increases, the segment does not undergo a radial reduction (in other terms, it does not shorten within space). This time, both the segment and the corresponding space undergo a radial contraction (the segment shortens together with space), whose size is unknown, and the surrounding spatial lattice, whose integrity must be in any case preserved, results in being deformed, but we don't know how this happens.

If we suppose that the segment (that represents the singularity) lies along the fourth axis, from (1), by setting equal to zero, one at a time, $x_{1}, x_{2}$ and $x_{3}$, we immediately obtain the following three-dimensional scenarios:

$$
\begin{aligned}
& x_{4,1}^{2}+x_{2}^{2}+x_{3}^{2} \leq R_{S}^{2} \\
& x_{1}^{2}+x_{4,2}^{2}+x_{3}^{2} \leq R_{S}^{2} \\
& x_{1}^{2}+x_{2}^{2}+x_{4,3}^{2} \leq R_{S}^{2}
\end{aligned}
$$

Very evidently, if we take into consideration one among the scenarios we have just obtained, the procedure previously discussed (the creation of the singularity) is equivalent to concentrating along the segment, bordered by $C$ and $O$, the mass of the spherical sector, whose amplitude is equal to $2 \chi_{\max }$. As previously highlighted, the new radial extension of the segment (that represents the singularity) is unknown, as well as the shape of the deformed spatial lattice. In other terms, we are forced to carry out some hypotheses. Let's denote with $r$ the radial coordinate of a generic point of the warped surface. Now, let's suppose that, notwithstanding the deformation of the spatial lattice induced by the mass, if the angular distance between whatever couple of points does not vary, the corresponding measured distance remains constant. In order to obtain the above, we must impose the following:

$$
\begin{gathered}
R_{S}=\sqrt{\left(\frac{d r}{d \chi}\right)^{2}+r^{2}} \\
2 \frac{d r}{d \chi} \frac{d^{2} r}{d \chi^{2}}+2 r \frac{d r}{d \chi}=2 r^{\prime} r^{\prime \prime}+2 r r^{\prime}=0
\end{gathered}
$$

The foregoing equation is characterized by the underlying general solution:

$$
r=K_{1} \cos \chi+K_{2} \sin \chi
$$

If we consider the range $\left[0, \chi_{\max }\right]$, with $\chi_{\max }$ such that $r\left(\chi_{\max }\right)=R_{s}$ and $r^{\prime}\left(\chi_{\max }\right)=0$, we obtain: 


$$
r(\chi)=R_{S} \cos \left(\chi_{\max }-\chi\right)
$$

The previous outcome is depicted in Figure 2.

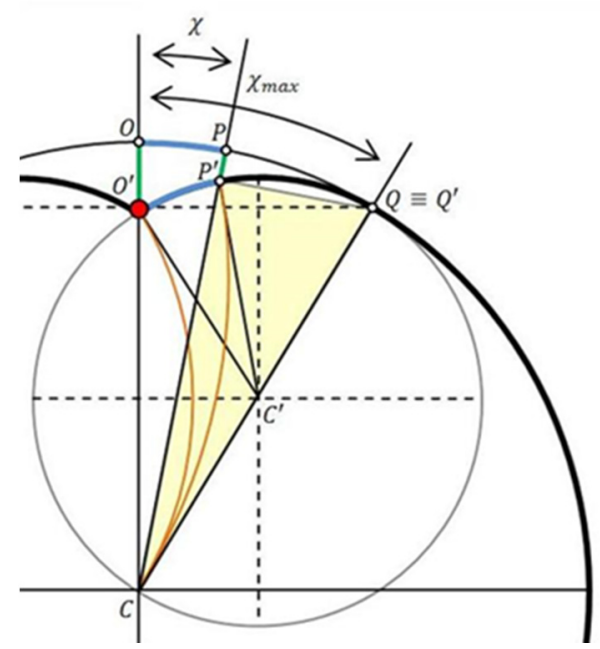

Figure 2. Gravitational Singularity and Warped Space

Figure 2 qualitatively shows how space results in being deformed due to a gravitational singularity (perceived as being placed in $O^{\prime}$ ). At the beginning, when mass is evenly spread, the origin coincides with $O$. If we concentrate in $O$ (actually along the segment bordered by $C$ and $O$ ) the mass contained in the spherical sector (actually a hyperspherical sector) whose amplitude is equal to $2 \chi_{\max }$, space undergoes a contraction. The new origin is placed in $O$ ', and the surrounding space is symmetrically warped. The initial radial coordinate of a generic point $P$ (actually its initial radial extension), is represented by the segment bordered by $C$ and $P$. The angular distance is denoted by $\chi$. The final coordinate (actually the final radial extension), represented by the segment bordered by $C$ and $P^{\prime}$, is shorter than the initial one, and its value is provided by (16). The proper radius does not undergo any modification whatsoever: the arc bordered by $O$ and $P$, in fact, is evidently equal to the one bordered by $O$ ' and $P$ '.

If we denote with $x$ the reduced "flat coordinate" (the reduced forecast radius), we have:

$$
x=r \sin \chi=R_{S} \sin \chi \cos \left(\chi_{\max }-\chi\right)
$$

Moreover, with obvious meaning of the notation, we can immediately write:

$$
\begin{gathered}
r_{\text {min }}=r(0)=R_{S} \cos \chi_{\text {max }} \\
\Delta r(\chi)=R_{s}-r(\chi)=R_{S}\left[1-\cos \left(\chi_{\text {max }}-\chi\right)\right] \\
\Delta r_{\text {max }}=\Delta r(0)=R_{S}\left(1-\cos \chi_{\text {max }}\right)
\end{gathered}
$$

If we denote with $M$ the mass of the ball (actually the 4-ball with which we identify our Universe), and with $M_{\chi}$ the one contained in the spherical sector (actually a hyper-spherical sector) whose amplitude is equal to $2 \chi$, we can write, taking into account (20), the following:

$$
\begin{gathered}
\frac{M_{\chi}}{M}=\frac{\Delta r_{\text {max }}}{R_{s}}=\frac{R_{s, \chi}}{R_{s}} \\
R_{s, \chi}=\Delta r_{\text {max }}=R_{S}\left(1-\cos \chi_{\text {max }}\right)
\end{gathered}
$$

In other terms, we can state that, if we concentrate in $O$ (actually along the material segment whose edge coincides with $O$ ) the mass contained in the spherical sector (actually a hyper-spherical sector) whose amplitude is equal to $2 \chi$, the radial coordinate of $O$ will undergo a reduction (actually the material segment will undergo a contraction) 
whose size is equal to the Schwarzschild radius of $M_{\chi}$. The hypothesized scenario is qualitatively portrayed in the following figure, where the singularity (as we can perceive it) coincides with the point $O^{\prime}$.

The outcomes just obtained are qualitatively depicted in Figure 3.

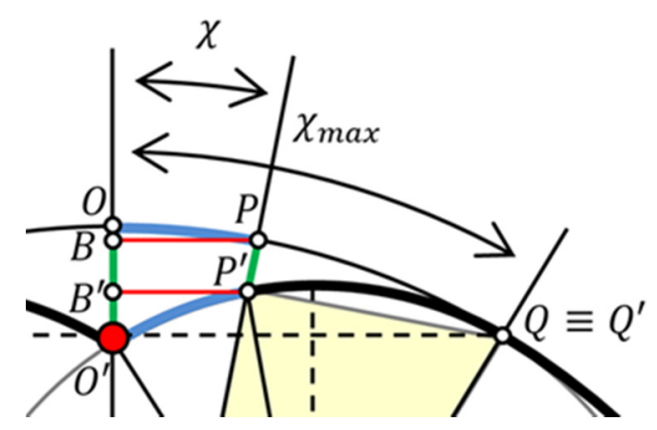

Figure 3. Gravitational Singularity and Warped Space (particular)

Figure 3 shows once again how the singularity, perceived as being placed in $O^{\prime}$, does not influence the measured distance (the proper radius). The arc bordered by $O$ and $P$, as previously underlined, is evidently equal to the one bordered by $O$ ' and $P^{\prime}$ '. On the contrary, the "flat coordinate" (the forecast radius) undergoes a reduction. The segment bordered by $B$ and $P$ represents the forecast radius $(X)$ when matter is evenly spread; the segment bordered by $B^{\prime}$ and $P$ represents the reduced forecast radius $(x)$.

\section{Vacuum Field solution (non-rotating and non-charged "black hole")}

Now, let's suppose that the whole available mass may be concentrated in $O$. In the light of the qualitative model we have just built, taking into account (16) and (17), setting $\chi_{\max }=\pi / 2$, we can write:

$$
\begin{gathered}
r=R_{S} \sin \chi \\
x=r \sin \chi=R_{S} \sin ^{2} \chi
\end{gathered}
$$

The scenario is qualitatively portrayed in Figure 4.

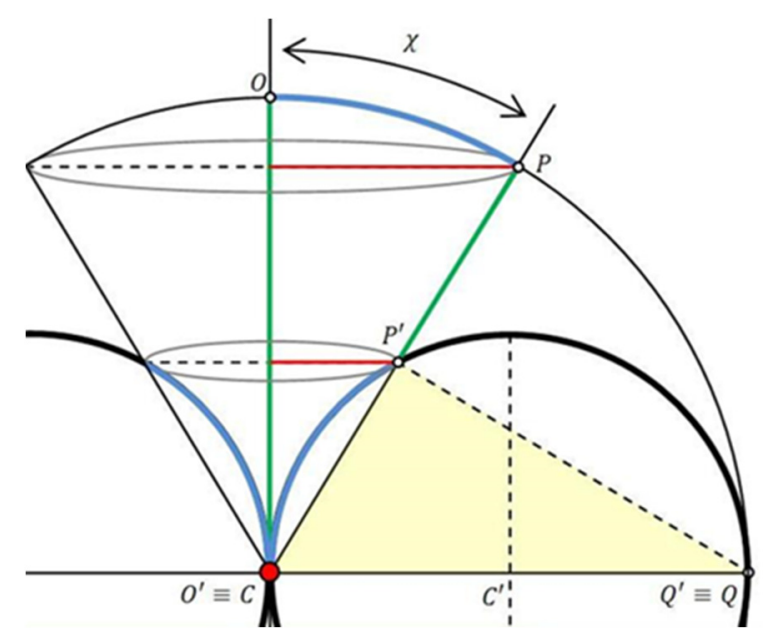

Figure 4. Non-Rotating and Non-Charged "Black Hole"

Figure 4 qualitatively depicts the case in which all the mass is concentrated in one single point. The origin, initially placed in $O$, coincides with the center of the ball (actually a 4-ball) with which we identify the Universe. 
As previously observed, once fixed the angular distance, the proper radius (the measured one) will remain the same, but the flat coordinate (and, consequently, the corresponding circumference) will undergo a reduction. Consequently, a test particle will take less time than forecast to draw a complete orbit around the singularity.

Now, let's suppose that we are not disposed to accept such a situation. More precisely, let's imagine that, instead of admitting that the orbit drawn by the test particle has undergone a contraction, we prefer to hypothesize that, due to the gravitational source, time has started slowing down. As a consequence, we may write:

$$
d t_{\chi}=\sin \chi d t_{0}
$$

Clearly, we have denoted with $t_{0}$ the absolute time, and with $t_{\chi}$ the "local" time. By virtue of this interpretation, taking into account the fact that, coherently with the model, a light impulse takes the same time, with or without gravitational singularity, to cover whatever distance (once fixed $\chi$ ), we are forced to modify the value of the proper radius. The reason is very simple. On the one hand, the speed of light cannot be influenced by the singularity; on the other hand, we forcefully postulate that, due to the gravitational source, time starts slowing down (in other terms, we refuse to admit that the orbit undergoes a contraction). As a consequence, in order to keep the speed of light constant, we have to imagine that the proper radius undergoes a dilation. To summarize the results up to now obtained, we can state that, apparently, if we concentrate matter in a point, time and space (the proper radius) undergo a dilation. Similarly, we can state that, apparently, if we remove mass from a gravitational source, time and space (the proper radius) undergo a contraction. The length of whatever orbit remains (apparently) constant.

Now, let's carry out a reparameterization of (23). We may write:

$$
r^{*}=R^{*} \sin \chi
$$

Obviously, we will avoid considering the banal case in which the derivative of $R^{*}$ is null. We have:

$$
\begin{gathered}
\frac{d r^{*}}{d \chi}=\frac{d R^{*}}{d \chi} \sin \chi+R^{*} \cos \chi \\
r^{* 2}+\left(\frac{d r^{*}}{d \chi}\right)^{2}=R^{* 2}+\left(\frac{d R^{*}}{d \chi}\right)^{2} \sin ^{2} \chi+2 R^{*} \frac{d R^{*}}{d \chi} \sin \chi \cos \chi \\
r^{* 2}+\left(\frac{d r^{*}}{d \chi}\right)^{2}=R^{* 2}+\left(\frac{d R^{*}}{d \chi}\right)^{2}\left[\sin ^{2} \chi+\left(\frac{2 R^{*} \tan \chi}{\frac{d R^{*}}{d \chi}}\right) \cos ^{2} \chi\right]
\end{gathered}
$$

Since the proper radius must actually remain the same, in spite of the fact that all the mass is concentrated in a single point, we have to impose the following:

$$
d l_{r^{*}}=\sqrt{r^{* 2}+\left(\frac{d r^{*}}{d \chi}\right)^{2}} d \chi=\sqrt{R^{* 2}+\left(\frac{d R^{*}}{d \chi}\right)^{2}} d \chi=d l_{R^{*}}
$$

Consequently, from (29), to satisfy the previous condition, we must impose the following:

$$
2 \tan \chi d \chi=\frac{d R^{*}}{R^{*}}
$$

Finally, if we impose that $R^{*}(0)=R_{s}$, we obtain:

$$
\begin{gathered}
R^{*}=\frac{R_{s}}{\cos ^{2} \chi} \\
d R^{*}=2 R_{s} \frac{\sin \chi}{\cos ^{3} \chi} d \chi \\
\sin \chi=\sqrt{1-\frac{R_{s}}{R^{*}}}
\end{gathered}
$$


Taking into account (4), the "flat coordinate" can be now written as follows:

$$
X^{*}=R^{*} \sin \chi=R_{s} \frac{\sin \chi}{\cos ^{2} \chi}=r^{*}
$$

The measure of the forecast (great) circumference is provided by the following banal identity:

$$
C_{X^{*}}=2 \pi r^{*}
$$

Taking into account (24), the reduced "flat coordinate" can now be written as follows:

$$
x^{*}=r^{*} \sin \chi=R_{S} \tan ^{2} \chi=R^{*}-R_{S}
$$

Far from the gravitational source (this is an absolutely fundamental condition), we have:

$$
x^{*} \gg R_{S} \rightarrow x^{*} \cong R^{*}
$$

As a consequence, for the reduced (great) circumference we can write:

$$
C_{x^{*}}=2 \pi\left(R^{*}-R_{S}\right) \cong 2 \pi R^{*}
$$

When all the mass is concentrated in a single point (singularity), considering time as being absolute and accepting the fact that the radius of the orbit is provided by (39), we may write:

$$
d s_{x^{*}}^{2}=c^{2} d t^{2}-d l_{r^{*}}^{2}-R^{* 2}\left(d \theta^{2}+\sin ^{2} \theta d \varphi^{2}\right)
$$

For the initial scenario (no singularity, mass evenly spread), considering time as being absolute and accepting the fact that the radius of the orbit undergoes a dilation, we may write the following distance:

$$
d s_{X^{*}}{ }^{2}=c^{2} d t^{2}-d l_{R^{*}}^{2}-\frac{R^{* 2}}{\sin ^{2} \chi}\left(d \theta^{2}+\sin ^{2} \theta d \varphi^{2}\right)
$$

Obviously, since the proper radius remains constant, we have:

$$
d l_{R^{*}}=d l_{r^{*}}=\frac{2 R_{s}}{\cos ^{3} \chi} \sqrt{1+\frac{1}{4 \tan ^{2} \chi}} d \chi
$$

Now, starting from (40), let's return to the initial scenario. In the light of the forceful interpretation we have previously discussed, let's suppose that the length of the orbit remains constant, and that time undergoes a contraction as the proper radius does. Bearing in mind that the speed of light is constant, from (40) we obtain:

$$
d s_{X^{*}}^{2}=c^{2} \frac{d t^{2}}{\sin ^{2} \chi}-\sin ^{2} \chi d l_{R^{*}}^{2}-R^{* 2}\left(d \theta^{2}+\sin ^{2} \theta d \varphi^{2}\right)
$$

We can carry out the underlying positions:

$$
\begin{gathered}
\frac{d t}{\sin \chi}=d t^{*} \\
\sin \chi d l_{R^{*}}=d l^{*}
\end{gathered}
$$

It is very easy to verify that

$$
\lim _{\chi \rightarrow \pi / 2} \frac{d l_{R^{*}}}{d R^{*}}=\lim _{\chi \rightarrow \pi / 2} \frac{d l_{r^{*}}}{d R^{*}}=\lim _{\chi \rightarrow \pi / 2} \frac{d l^{*}}{d R^{*}}=1
$$

Taking into account the previous equation, we can write:

$$
x^{*} \gg R_{S} \rightarrow d l^{*} \cong d l_{R^{*}}=d l_{r^{*}} \cong d R^{*}
$$


Finally, far from the origin (that previously was the source), we can write the following approximated "flat" metric:

$$
d s_{X^{*}}{ }^{2}=c^{2} d t^{* 2}-d R^{* 2}-R^{* 2}\left(d \theta^{2}+\sin ^{2} \theta d \varphi^{2}\right)
$$

Now, if we concentrate all the mass in the origin, "pretending" that the length of the orbit will remain the same, and that time and proper radius will undergo a dilation, we may write the following "Schwarzschild-like" metric:

$$
d s_{x^{*}}{ }^{2}=c^{2}\left(1-\frac{R_{s}}{R^{*}}\right) d t^{* 2}-\frac{d R^{* 2}}{1-\frac{R_{s}}{R^{*}}}-R^{* 2}\left(d \theta^{2}+\sin ^{2} \theta d \varphi^{2}\right)=d s^{* 2}
$$

Referring to the metric tensor, we have:

$$
\begin{gathered}
g_{00}=1-\frac{R_{s}}{R^{*}}=\sin ^{2} \chi \\
g_{00} g_{11}=-1
\end{gathered}
$$

The previous identity is evidently satisfied for each of the distances we have written up until now, since it simply expresses the fact that the speed of light must remain constant.

Obviously, for the proper radius, denoted by $R_{p}\left(R^{*}\right)$, we can now write the following:

$$
R_{p}\left(R^{*}\right)=\int_{R_{s}}^{R^{*}} \sqrt{-g_{11}} d R^{*}=\int_{R_{S}}^{R^{*}} \frac{d R^{*}}{\sqrt{1-\frac{R_{s}}{R^{*}}}} d R^{*}=\sqrt{R^{*}\left(R^{*}-R_{S}\right)}+R_{S} \ln \left(\frac{\sqrt{R^{*}}+\sqrt{R^{*}-R_{S}}}{R_{S}}\right)
$$

Making explicit the Schwarzschild radius, we can write (49) as follows:

$$
d s^{* 2}=c^{2}\left(1-\frac{2 G M}{c^{2} R^{*}}\right) d t^{* 2}-\frac{d R^{* 2}}{1-\frac{2 G M}{c^{2} R^{*}}}-R^{* 2}\left(d \theta^{2}+\sin ^{2} \theta d \varphi^{2}\right)
$$

It is fundamental to underline how the real Schwarzschild solution (Schwarzschild, 1919) does not acquire the form of (53). If we want to maintain Schwarzschild's original notation, in fact, we have the following:

$$
d s^{2}=c^{2}\left(1-\frac{\alpha}{R}\right) d t^{* 2}-\frac{d R^{2}}{1-\frac{\alpha}{R}}-R^{2}\left(d \theta^{2}+\sin ^{2} \theta d \varphi^{2}\right)
$$

It is worth highlighting how, although $\alpha$ represents a constant that depends on the mass of the singularity, Schwarzschild himself did not specify its value. For coordinate $R$, moreover, his position is the following:

$$
R=\left(r^{3}+\alpha^{3}\right)^{1 / 3}
$$

Obviously, $r$ represents the "flat coordinate". If we denote with $x, y$ and $z$ the rectangular coordinates, we have:

$$
r=\sqrt{x^{2}+y^{2}+z^{2}}
$$

It is worth underlining how (55) does not represents the only possible position. This assertion can be easily verified, for example, by analyzing the solutions proposed by Droste (Droste, 1917), Hilbert (Antoci, 2004), and Brillouin (Brillouin, 1923).

From (54), by setting $\alpha$ equal to zero, we immediately obtain the following "flat" metric:

$$
d s^{2}=c^{2} d t^{2}-d r^{2}-r^{2}\left(d \theta^{2}+\sin ^{2} \theta d \varphi^{2}\right)
$$

\section{4. "Weak Field" approximation}

We have elsewhere discussed (Cataldo, 2017) how, due to the mass - energy equivalence (Einstein, 1916), a test particle, whose radial coordinate is equal to $r$, is forced to move with a speed, denoted by $v$, whose value, taking into account (23), is provided by the following equation: 


$$
v=c \sqrt{1-\left(\frac{r}{R_{s}}\right)^{2}}=c \cos \chi
$$

Since the motion is related, in our case, to the deformation of the spatial lattice, speed may be imagined as being characterized by two components, denoted by $v_{I}$ and $v_{I I}$. We have:

$$
\begin{gathered}
v=\sqrt{{v_{I}^{2}+v_{I I}^{2}}^{2}} \\
v_{I}=\frac{1}{2} c \sin (2 \chi) \\
v_{I I}=c \cos ^{2} \chi
\end{gathered}
$$

The two components of speed are qualitatively depicted in Figure 5.

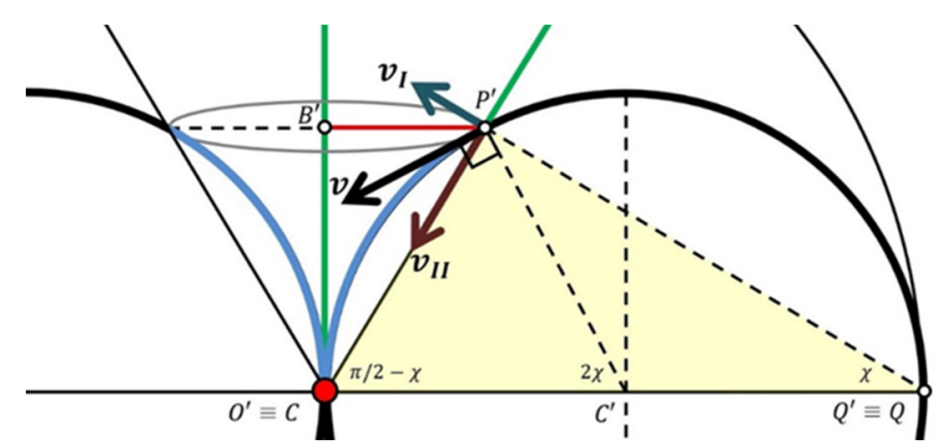

Figure 5. Components of the Speed of a Test Particle

Figure 5 shows how, when a test particle approaches the singularity, the value of $v_{I}$ decreases while, on the contrary, the value of $v_{I I}$ increases. It is commonly said that, in approaching the singularity, the space-like geodesics become time-like, and vice-versa. In our case, the above-mentioned interpretation is not correct, since the radial coordinate is nothing but the extension of a material segment, that we perceive as being a material point (the test particle), along the further spatial dimension herein postulated. The straight-line segment bordered by $C$ (that evidently coincides with $O^{\prime}$ ) and $P^{\prime}$ represents the radial extension of the particle, the one bordered by $B^{\prime}$ and $P^{\prime}$ represents the reduced "flat coordinate" (the radius of the reduced circumference).

The energy needed to produce the motion (Einstein, 1916), making explicit the so-called Lorentz factor (Lorentz, 1904), can be written as follows:

$$
\Delta E=m c^{2}(\gamma-1)=m c^{2}\left[\frac{1}{\sqrt{1-\left(\frac{v}{c}\right)^{2}}}-1\right]
$$

Obviously, we can imagine that the energy in question may be provided by a gravitational field (the coordinate of the particle depends on the shape of the spatial lattice). By virtue of (8) and (58), far from the source, we obtain:

$$
v \ll c \rightarrow \Delta E \cong \frac{1}{2} m v^{2}=\frac{G M m}{R_{S}} \cos ^{2} \chi
$$

If we denote with $\phi$ the potential, we can write, far from the source, the following pseudo-Newtonian relation:

$$
\phi=\frac{G M}{\frac{R_{S}}{\cos ^{2} \chi}}=\frac{G M}{R^{*}}
$$

From the foregoing equation, taking into account (34) and (50), we have: 


$$
\begin{gathered}
\frac{2 \phi}{c^{2}}=\frac{2 G M}{c^{2} R^{*}}=\frac{R_{s}}{R^{*}}=1-\sin ^{2} \chi \\
\phi=\frac{1}{2} c^{2}\left(1-g_{00}\right)
\end{gathered}
$$

The previous equation coincides with the so-called "weak field" expression for the gravitational potential.

\section{Final Remarks and Conclusions}

The coordinate $R^{*}$, that appears both in the metric and at the denominator of the pseudo-Newtonian relation we have obtained for the gravitational potential, does not represent a real distance nor a real radius of curvature. In fact, it is clear enough how the expression of $R^{*}$ arises from a banal reparameterization, by means of which we are able to write a metric that, far from the origin, may approximatively describe, what is more, not a void space, peculiar to Special Relativity, but rather a Universe evenly filled with matter. According to the simple qualitative model herein proposed, the so-called Black Holes may not exist. Consequently, there is no reason to keep on concretely considering the existence of an alleged "event horizon". On the one hand, in fact, the minimum value that can be assumed by the coordinate $R^{*}$ is equal to the Schwarzschild radius. On the other hand, when it happens, both the proper radius and the forecast one are equal to zero: in other terms, we are exactly placed in correspondence of the singularity. Once accepted the fact that time is not influenced by gravity, to obtain something similar to what is commonly considered as being a Black Hole, we should concentrate in a single point all the mass contained in the entire Universe. In this case, if a test particle were to reach the singularity, its escape velocity would equate that of light: however, once again, time would keep on flowing in the usual manner. In this article, we have discussed in terms of warped space: actually, if it were so, we would be forced to admit that the shape of the Universe can be modified with respect to something else, taken as a reference. In the light of the previous remark, we should rather imagine that the value of space may change. In order to validate such an assertion, it would have been necessary to start a discussion that, without any doubt whatsoever, would have gone far beyond the aim of this paper, since the topic involves the concept of dimensional thickness, elsewhere expounded (Cataldo, 2016b), as well as phenomena such as the so-called gravitational redshift. Moreover, the discussion has been carried out taking for granted the global symmetry elsewhere postulated (Cataldo, 2016c).

\section{References}

Antoci, S. (2004). David Hilbert and the origin of the "Schwarzschild solution". Meteorological and Geophysical Fluid Dynamics (a book to commemorate the centenary of the birth of Hans Ertel), W. Schroder Editor, Science Edition, Bremen. Retrieved from https://arxiv.org/abs/physics/0310104

Brillouin, M. (1923). Les points singuliers de l'univers d'Einstein. Journal de Physique et le Radium, 4(1), 43-48 (The singular points of Einstein's Universe, translated by Salvatore Antoci, 2000). Retrieved from https://arxiv.org/pdf/physics/0002009.pdf

Cataldo C. (2016b). From the Oscillating Universe to Relativistic Energy: a Review. Journal of High Energy Physics, Gravitation and Cosmology, 3, 68-77. http://dx.doi.org/10.4236/jhepgc.2017.31010

Cataldo, C. (2016a). Faster than Light: again on the Lorentz Transformations. Applied Physics Research, 8(6), 1724. http://dx.doi.org/10.5539/apr.v8n6p17

Cataldo, C. (2016c). Effects of a Global Symmetry on the Observation of Astronomical Objects. Applied Physics Research, 8(5), 75-80. http://dx.doi.org/10.5539/apr.v8n5p75

Cataldo, C. (2017). From General Relativity to A Simple-Harmonically Oscillating Universe, and Vice-Versa: A Review. Applied Physics Research, 9(1), 86-92. http://dx.doi.org/10.5539/apr.v9n1p86

Droste, J. (1917). The field of a single centre in Einstein's theory of gravitation and the motion of a particle in that field. Royal Netherlands Academy of Arts and Sciences (KNAW), Proceedings, 19 I, 197-215. Retrieved from http://www.dwc.knaw.nl/DL/publications/PU00012346.pdf

Einstein, A. (1916). Relativity: The Special and General Theory (translated by R. W. Lawson, 1920). Henry Holt and Company, New York. Retrieved from https://archive.org/details/cu31924011804774

Friedmann A. (1922). Über die Krümmung des Raumes. Zeitschrift für Physik, 10, 377-386. English Translation (1999) On the Curvature of Space. General Relativity and Gravitation, 31(12), 1991-2000. Retrieved from http://www.ymambrini.com/My_World/History_files/Friedman_1922.pdf 
Harrison, E. R. (1967). Classification of uniform cosmological models. Monthly Notices of the Roy. Astron. Soc., 137, 69-79. Retrieved from http://paperity.org/p/39522229/classification-of-uniform -cosmological-models

Lorentz, H. A. (1904) Electromagnetic phenomena in a system moving with any velocity smaller than that of light. Proceedings of the Royal Netherlands Academy of Arts and Sciences, 6, 809-831. Retrieved from http://www.orgonelab.org/EtherDrift/Lorentz1904.pdf

Schwarzschild K. (1919). Über das Gravitationsfeld eines Massenpunktes nach der Einsteinschen Theorie. Sitzungsberichte der Deutschen Akademie der Wissenschaften zu Berlin, 189-196 (On the Gravitational Field of a Mass Point according to Einstein's Theory, translated by Antoci S. and Loinger A., 1999). Retrieved from http://zelmanov.ptep-online.com/papers/zj-2008-03.pdf

\section{Copyrights}

Copyright for this article is retained by the author(s), with first publication rights granted to the journal.

This is an open-access article distributed under the terms and conditions of the Creative Commons Attribution license (http://creativecommons.org/licenses/by/4.0/). 